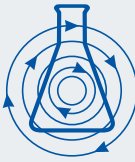

\title{
New Brunswick
}

an eppendorf company

\section{New benchtop bioreactor uses presterilized, prevalidated, single-use vessels to simplify cell culture}

New Brunswick Scientific has introduced a benchtop cell culture bioreactor that combines the benefits of single-use technology with the trusted performance and true scalability of a stirred-tank design. CelliGen ${ }^{\mathrm{TM}}$ BLU eliminates the need for autoclaving and cleaning, reduces validation requirements, provides rapid turnaround between runs, minimizes startup costs and significantly reduces the risk of contamination because the vessel is used once and then discarded. A compact controller enables advanced process management for research or manufacturing.

Single-use, stirred-tank vessels

CelliGen BLU (Fig. 1) features interchangeable, single-use, rigidwall, stirred-tank vessels in 5.0 and 14.0 liter total volume capacities. Vessels are delivered preassembled with pitched-blade impeller, porous microsparge and all the necessary tubing, filters and connectors; they are presterilized, ready for use right out of the box. All components in product contact are made of materials that meet USP class VI standards and have been tested for leachables and extractables, making these vessels appropriate for current good manufacturing practices (cGMP) environments.

Headplate penetrations are provided for a temperature sensor, $\mathrm{pH}$ and dissolved oxygen (DO) probes, three addition ports, harvest, exhaust, sample, overlay and gas sparge. A reusable heat blanket with a window cutout for viewing cultures is provided for maintaining temperature in the culture vessel. DO is monitored via a noninvasive, reusable polarographic probe. $\mathrm{pH}$ is monitored via a noninvasive optical $\mathrm{pH}$ probe and disposable fluorescence sensor.

\section{Advanced process control}

CelliGen BLU's control station includes NBS's powerful reactor process controller (RPC) firmware to provide advanced process management and monitoring capability. An adjustable-position, 15-inch industrial color touchscreen monitor makes it easy to set and monitor up to 32 process parameters and store up to ten recipes, as well as view up to eight parameters simultaneously on a user-customizable trend graph. Built-in security features include variable levels of control functionality for operators, supervisors and administrators, as well as passwords to prevent unauthorized users from making any changes to the system.

\section{Guozheng Wang, Wenying Zhang \& Rich Mirro}

New Brunswick Scientific, Edison, New Jersey, USA. Correspondence should be addressed to R.M. (bioinfo@nbsc.com).

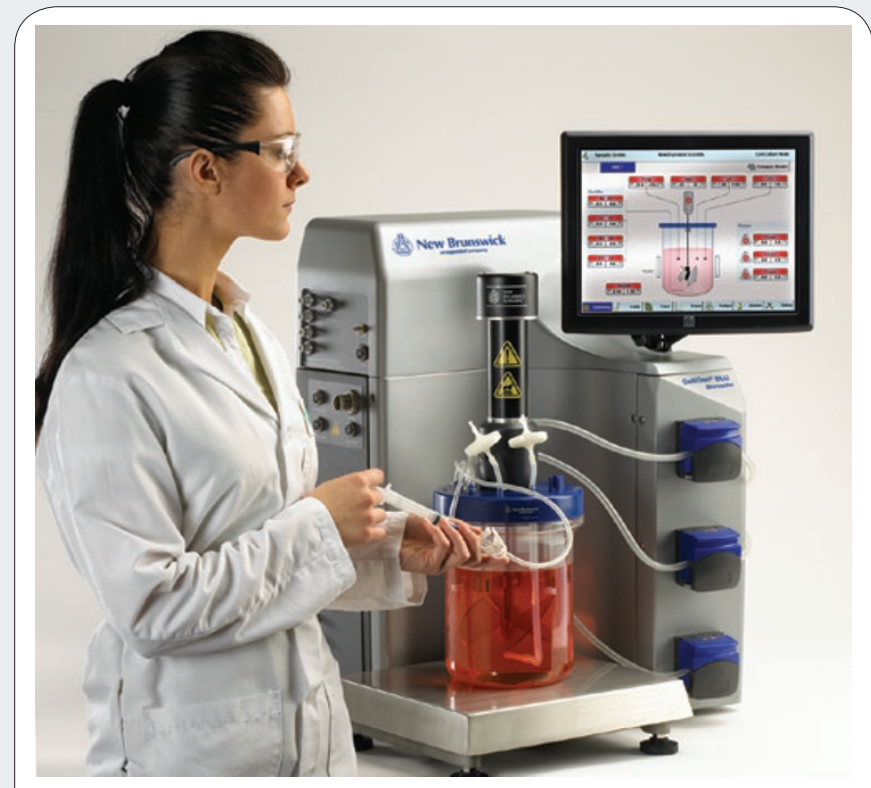

Figure 1 | The new CelliGen BLU cell culture bioreactor features single-use, stirred-tank vessels and an advanced process controller for growth of animal cells in CGMP-compliant research and production environments.

The compact control station includes three high-performance, assignable, fixed-speed pumps for additions and harvesting. Built-in controls are also provided for temperature, $\mathrm{pH}$, DO and three- or four-gas mixing. Up to four thermal mass flow controllers (TMFC) for fully independent gas control are available for sparging, and a gas overlay with TMFC or rotameter is available for independent control of gas entering the vessel headspace. An optional weight scale for measuring additions and volume by weight, as well as optional gas analyzers, BioCommand ${ }^{\circledR}$ supervisory control and data acquisition (SCADA) software, validation packages and more, enable customization to your needs. 
Table 1 | Comparison of $\mathrm{CHO}$ growth and viability achieved using three different bioreactor systems

\begin{tabular}{lccc}
\hline & \multicolumn{3}{c}{ CelliGen BLU bioreactor } \\
\cline { 2 - 4 } Day & $\begin{array}{c}\text { Total cells } \\
\left(\times 10^{6} \mathrm{ml}^{-1}\right)\end{array}$ & $\begin{array}{c}\text { Viable cells } \\
\left(\times 10^{6} \mathrm{ml}^{-1}\right)\end{array}$ & $\begin{array}{c}\text { Viability } \\
(\%)\end{array}$ \\
\hline 0 & 0.31 & 0.30 & 97.9 \\
1 & 0.69 & 0.68 & 97.1 \\
2 & 1.42 & 1.39 & 97.6 \\
3 & 2.57 & 2.51 & 97.6 \\
4 & 4.02 & 3.92 & 97.5 \\
5 & 5.70 & 5.55 & 97.3 \\
6 & 5.98 & 4.52 & 76.6 \\
7 & 6.71 & 3.21 & 47.8
\end{tabular}

\begin{tabular}{ccc}
\multicolumn{3}{c}{ CelliGen $\mathbf{3 1 0}$ bioreactor } \\
\hline $\begin{array}{c}\text { Total cells } \\
\left(\times 10^{6} \mathrm{ml}^{-1}\right)\end{array}$ & $\begin{array}{c}\text { Viable cells } \\
\left(\times 10^{6} \mathrm{ml}^{-1}\right)\end{array}$ & $\begin{array}{c}\text { Viability } \\
(\%)\end{array}$ \\
\hline 0.31 & 0.30 & 97.9 \\
0.64 & 0.62 & 96.5 \\
1.31 & 1.29 & 97.9 \\
2.47 & 2.41 & 97.8 \\
4.04 & 3.98 & 98.6 \\
5.46 & 5.39 & 98.7 \\
5.67 & 4.13 & 72.7 \\
6.25 & 3.12 & 49.7
\end{tabular}

\begin{tabular}{ccc}
\multicolumn{3}{c}{ Bag-and-rocker system } \\
\hline $\begin{array}{c}\text { Total cells } \\
\left(\times 10^{6} \mathrm{ml}^{-1}\right)\end{array}$ & $\begin{array}{c}\text { Viable cells } \\
\left(\times 10^{6} \mathrm{ml}^{-1}\right)\end{array}$ & $\begin{array}{c}\text { Viability } \\
(\%)\end{array}$ \\
\hline 0.31 & 0.30 & 97.9 \\
0.61 & 0.58 & 96.8 \\
1.33 & 1.30 & 97.6 \\
2.36 & 2.32 & 98.4 \\
3.89 & 3.83 & 98.5 \\
4.83 & 4.77 & 98.9 \\
5.46 & 3.67 & 67.3 \\
5.59 & 2.73 & 48.8
\end{tabular}

\section{Comparison to similar systems}

To prove the CelliGen BLU's ability to control growth conditions at set point as well as to produce high cell densities, researchers at New Brunswick Scientific's in-house laboratory compared growth of a Chinese hamster ovary (CHO) cell line (ATCC) using three bioreactor systems: the CelliGen BLU benchtop bioreactor with 5 liter totalvolume and 3.5 liter working-volume presterilized, single-use vessels; the CelliGen 310 benchtop autoclavable bioreactor with 5 liter totalvolume and 3.75 liter working-volume autoclavable vessels (NBS); and a bag-and-rocker style culture system with 10 liter total-volume and 5 liter working-volume presterilized, single-use bags.

The $\mathrm{CHO}$ line was preadapted to serum-free medium (CD CHO, Invitrogen).

Control parameters for all three systems were set as identically as possible. CelliGen BLU set points were as follows: temperature, $37^{\circ} \mathrm{C}$; pH, 7.0; dead-band, 0.10; DO, 40\%; agitation, 70-80 r.p.m.; gas overlay, $0.2-0.3$ standard liters per minute (SLPM) via four-gas mode for the entire run; and gas sparger using four-gas mode at 0-20 cubic centimeters per minute (CCM) via 'Cascade' control from the DO to 'Gasflo'

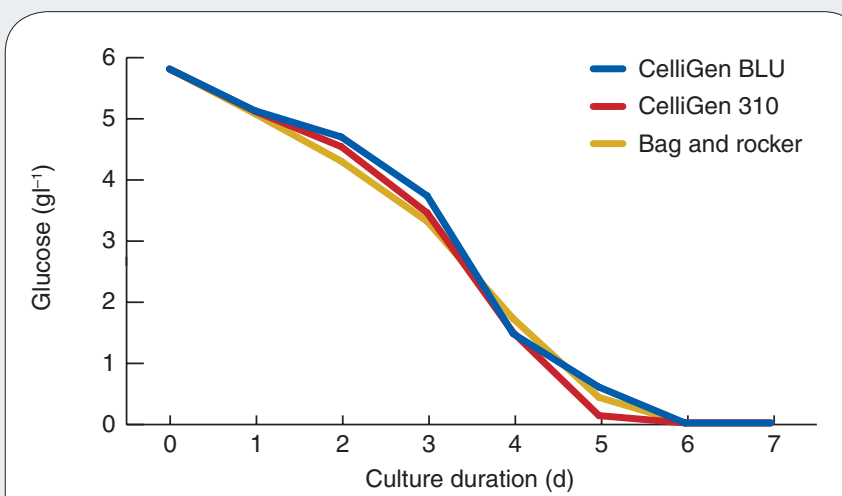

Figure 2 | Glucose concentration versus culture time for $\mathrm{CHO}$ cell cultures in three benchtop bioreactor systems. after 2 days of the run. CelliGen 310 set points were identical except for the following: agitation, 80-100 r.p.m.; gas overlay, 0.2-0.3 SLPM using four-gas mode for the first 2 days; and gas sparger at $0.1-0.20$ SLPM by four-gas mode after 2 days of the run. Bag-and-rocker bioreactor set points were chosen to be nearly equivalent to those used in the stirred-tank reactors: temperature, $37^{\circ} \mathrm{C} ; \mathrm{pH}, \sim 7.0$; DO, $40 \%$; rocking speed, 18 r.p.m.; rocking angle, $8^{\circ}$; and gas overlay at $0.1-0.3$ SLPM by air containing $0 \%-5 \% \mathrm{CO}_{2}$ based on the $\mathrm{pH}$.

Each of the batch runs lasted 7 days. Daily off-line measurements of glucose and lactate concentrations were read using a YSI 2700 analyzer (YSI Inc.), and cell density and cell viability were measured using a NucleoCounter (NBS).

The $\mathrm{CHO}$ cells in all three bioreactor systems grew steadily through day 5 of the 7-day run (Table 1), coinciding with availability of nutrients in the medium. By day 5 , a nutrient source, glucose, had become nearly depleted from the medium (Fig. 2). Cell viability in all three systems also remained high, at $96 \%$ or better, through day 5 . The maximum viable cell count attained in each system was as follows: $5.55 \times 106$ cells $\mathrm{ml}^{-1}$ by the CelliGen BLU, compared to $5.39 \times 10^{6}$ cells $\mathrm{ml}^{-1}$ for the autoclavable system and $4.77 \times 10^{6}$ cells ml$^{-1}$ for the bag-and-rocker system.

\section{Conclusion}

While no effort was made to optimize either the medium or the cell culture process control parameters in any study, these data demonstrate that the CelliGen BLU bioreactor is an efficient system for the culture of $\mathrm{CHO}$ cells, performs as well as traditional autoclavable stirred-tank bioreactors and can outperform bag-style systems. For a complete protocol with system setup or for more information on the CelliGen BLU, see http://www.nbsc.com/BLU/.

This article was submitted to Nature Methods by a commercial organization and has not been peer reviewed. Nature Methods takes no responsibility for the accuracy or otherwise of the information provided. 